\title{
Overcoming resistance to cancer immunotherapy
}

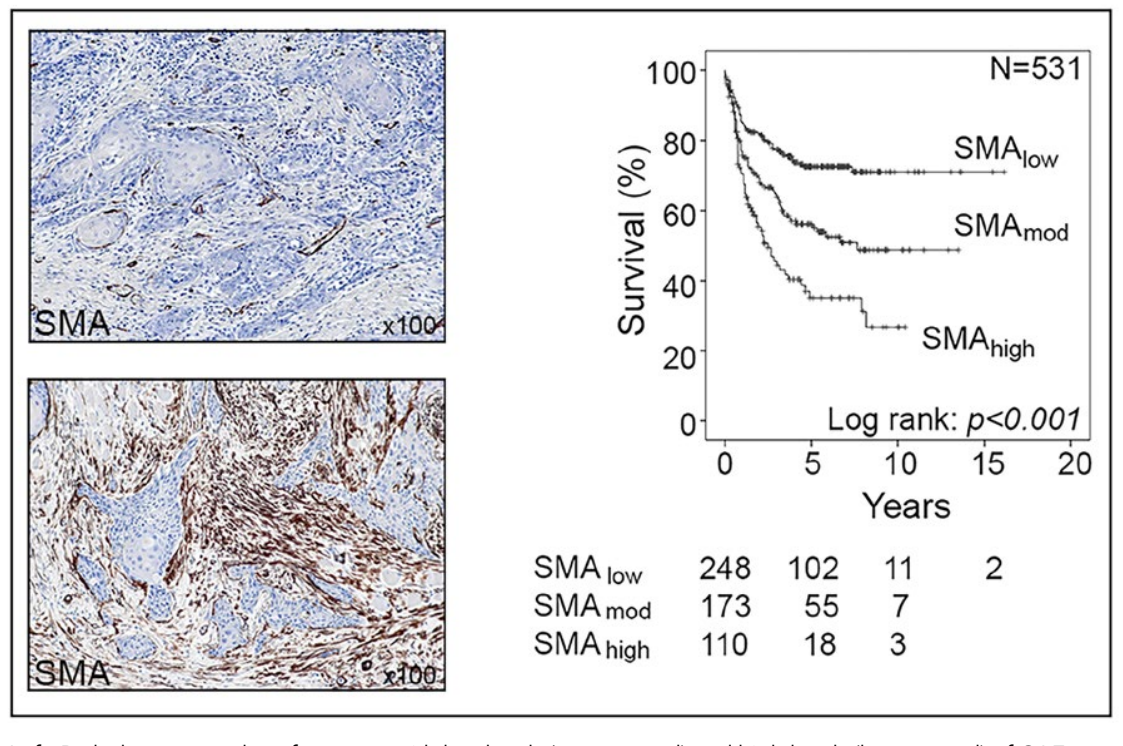

Left: Pathology examples of tumours with low levels (upper panel) and high levels (lower panel) of CAF
shown by inmunohistochemistry for the CAF Carker, smooth muscle actin (SMM). Right: The Kaplan-Meir
Sunvival curve shows how CAF levels significantly affect patient survival (head and neck cancer patients).

CAFs protect tumours from attack by the immune system by triggering the development of a defensive shield around the tumour.

and secrete extracellular matrix - a network of collagens and other proteins which produce scar tissue. Uncontrolled activation of fibroblasts results in tissue fibrosis, including organ fibrosis and atherosclerosis, and it is thought that around $45 \%$ of deaths are related to fibrosis in some form or anothe Cancerous cells also have the ability to "activate" fibroblasts, turning them into CAFs. These cells are found in lange numbers in many types of tumour in multiple different ways. For example, CAFs trigger cancers to grow more rapidly and spread, and recent studies have shown that CAF shield tumours from immune attack. A significant proportion of cancers are CAF-rich; these usually behave aggressively and are associated with poor patient survival.

In recent work, Professor Thomas and his team investigated exactly how CAFs regulate immunotherapy resistance and identified a therapy to overcome this. To do this, the researchers used a variety of experimental techniques, including mouse models specifically developed by reflect the CAF properties of human
(Genkyotex), a small-molecule NOX4 block the activity of $C A$ ss and increase the likelihood of success immunotherapy.

REVEALING THE ROLE OF CAFS The team discovered that CAFs function by specifically excluding a group of lymphocytes, $\mathrm{CD} 8^{+} T$ cells, from tumours (another type, $\mathrm{CD}^{+} \mathrm{T}$ cells, are able to enter). $C D 8^{+} \mathrm{T}$-cells are important effectors of the immune system and are responsible for killing cancer cells; most current immunotherapies depend on the ability of $\mathrm{CD} 8^{+} T$ cells to infiltrate tumours effectively. In tumours containing CAF, CD8 + T-cells mass; this exclusion edfect resumour mass; this exclusion effect resulted in checkpoint therapy.

Effectively, CAFs protect tumours from attack by the immune system by developing a defensive shield aroun the tumour. In the mouse models, artificially-created high levels of CAFs lymphocytes were unable to successfully penetrate the tumour. The team are currently investigating how CAF produce this effect, examining how CAF affect T-cell function and movement.

NOX4 - THE KEY TO NEUTRALISING CAFS? Until now, treatments that target CAFs

as part of cancer therapy have been
unsuccessful. This is likely due to the fact

playing a mPH Oxidase-4 playing a major role in activating and sustaining CAF, and the researchers
also tested a drug called Setanaxib

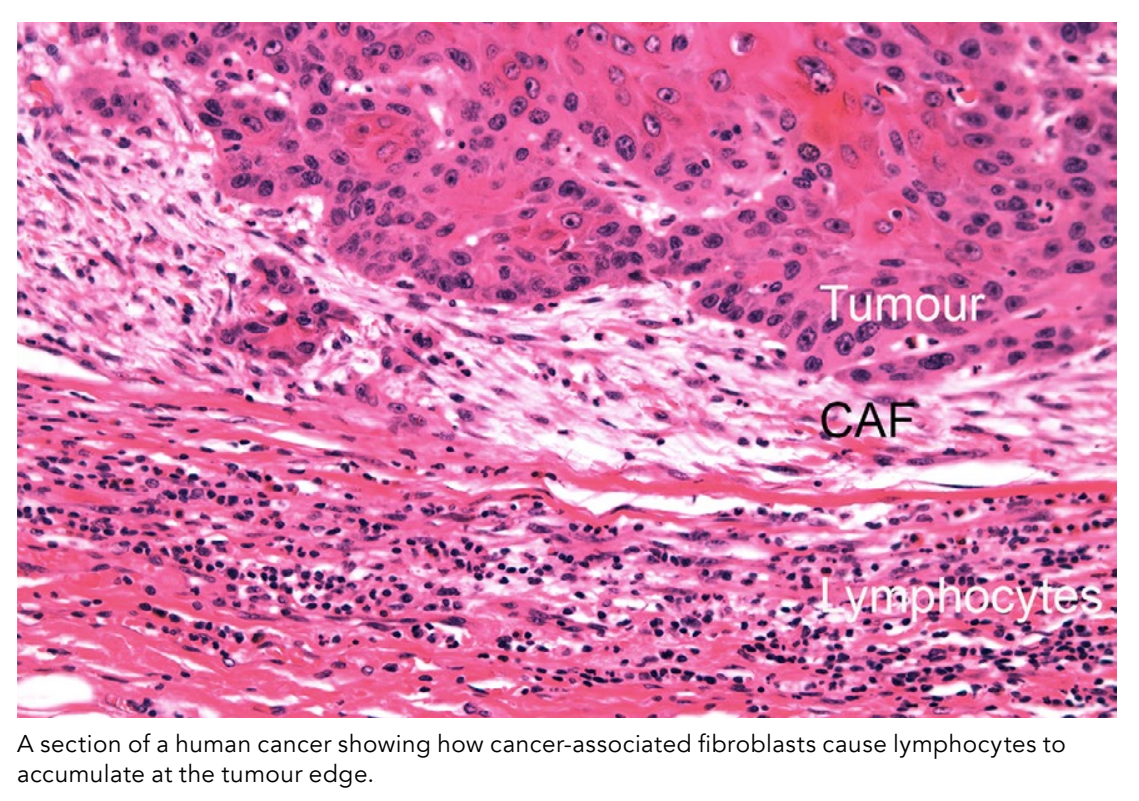

c|lPhotoss/Shuterstockcom directed against 'immune checkpoints' found on T-cells. Immune checkpoin 

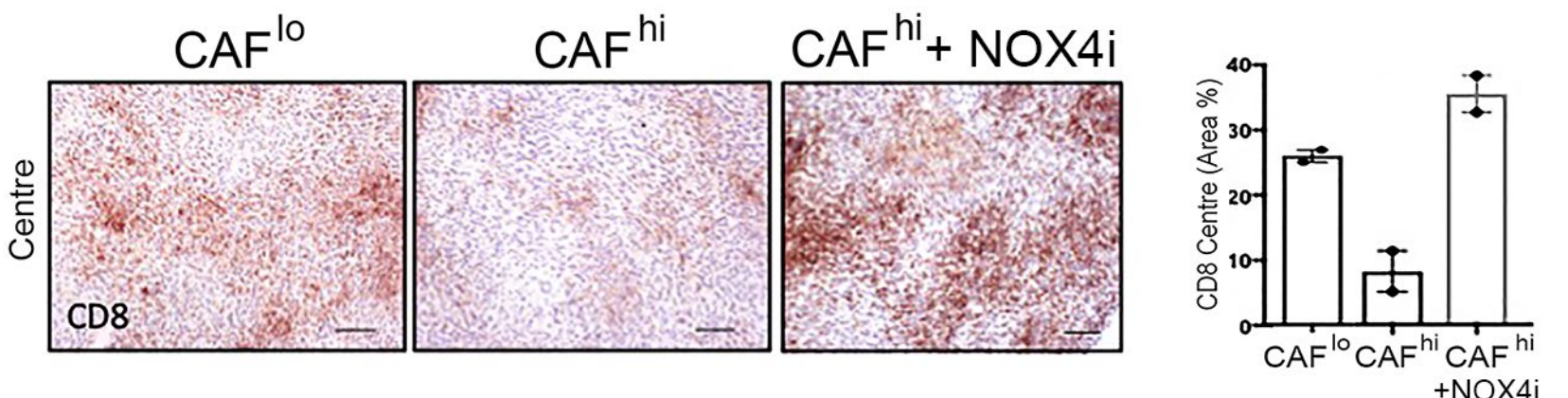

Immunochemistry of $\mathrm{CDD}^{+} \mathrm{T}$-cells (brown staining) in mouse tumours. The results show how increasing $\mathrm{CAF}$ numbers reduces $\mathrm{CD} 8^{+}-$-cells in tumours
(middlec

that not all CAFs are the same; in fact, they are a heterogeneous group of cells that share certain features, with some CAFs even shown to suppress tumour growth. Recent studies using single cell on CAF heterogeneity, and identified

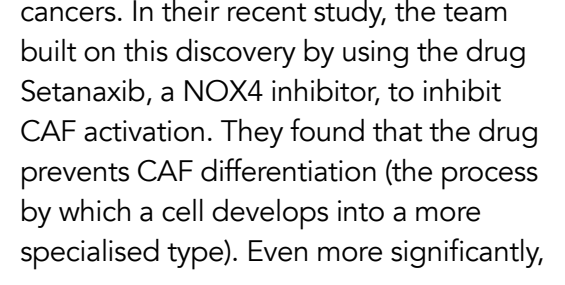

specialised type). Even more significantly

CAFs are not fixed in their final form, but need the continuous activity of NOX4 to maintain their cancer-associated state.

different CAF subpopulations, but up to this point, the lack of understanding of CAF phenotype, function and regulatory mechanisms have made targeting CAF difficult and resulted in a lack of specific therapeutic targets.

In previous work, Professor Thomas and his colleagues demonstrated th they discovered that Setanaxib can "normalise" previously-established CAFs, so that they behave more like a normal fibroblast.

In a critical step towards more successfu immunotherapy, the team showed that CAFs are not fixed in their final form, but need the continuous activity

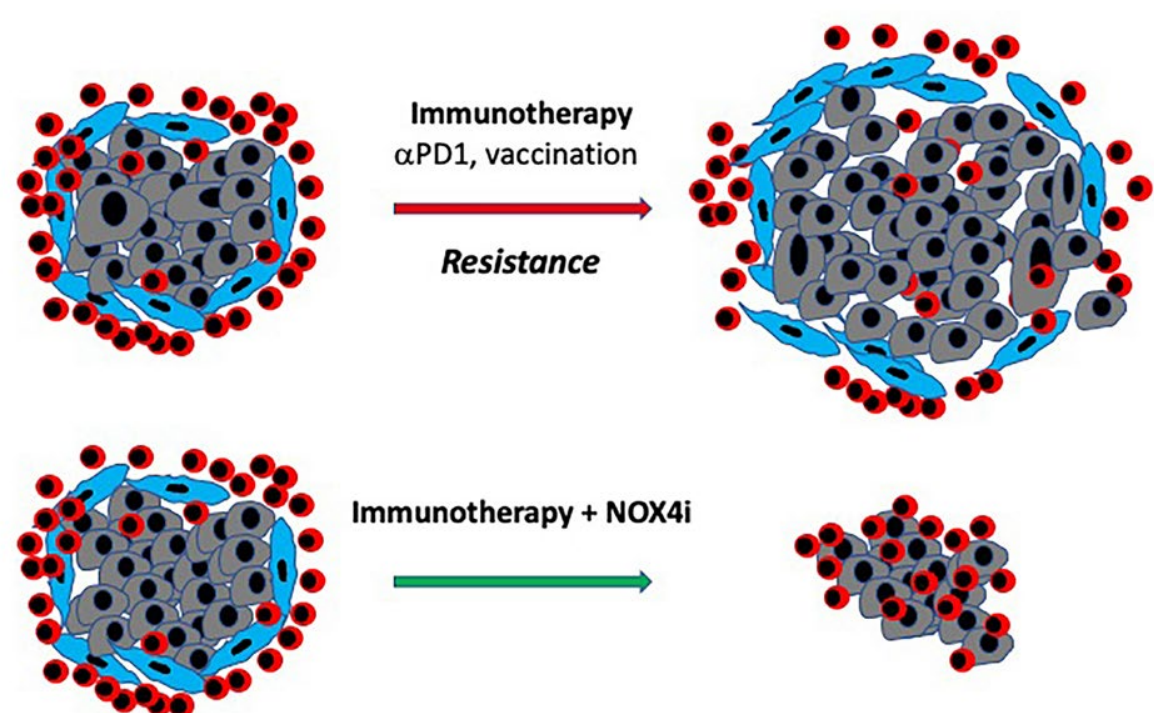

$2 \mathrm{CAF}$

- Tumour cell state. NOX4 inhibition was able to both prevent and reverse CAF formation in mouse tumours, triggering a fundamental change in the environmen surrounding the tumour. The tumourprotective power of CAFs was effectively Pliminated, allowing CD8 T-cells to

This finding was proven by the final

stage of the study: combining Setanaxib with several different currently-available immunotherapies including anti-tumour vaccination and anti-PD1 checkpoint therapy. The combination of Setanaxib with immunotherapy was able to promote the immunotherapy response CAF-laden tumours that were previously resistant to the treatment.

Setanaxib has several other advantages that make it an excellent choice for further investigation as a cancer treatment: it has already been tested in several clinical trials for fibrotic NCT03226067, NCTO2010242) and known to be extremely safe it is also simple to administer, and effective, whe taken orally.

TOWARDS MORE SUCCESSFUL IMMUNOTHERAPY Despite the exciting potential of immunotherapy, CAF-rich tumours respond poorly to current treatments, resulting in immunotherapy resistance in a large majority of patients. Professor Thomas and his colleagues believe that targeting CAFs with NOX4 inhibitors, as successfully demonstrated in their research, offers hope for more effective treatment of a wide range of cancers, In the relatively near future, these discoveries could benefit many cancer patients, some of whom may currently
have few alternative treatment options.

\section{Behind the Research}

\section{Professor Gareth Thomas}

E: g.thomas@soton.ac.uk T: $+44(0) 7845361124$

\section{Research Objectives}

The Gareth Thomas laboratory focuses on the role of cancer-associated fibroblasts in cancer, understanding can be targeted as part of cancer therapy-

\section{Detail}

Bio

Gareth Thomas is Professor of Experimental Pathology at the University of Southampton and a Consultant in Cellular Pathology.

Funding

Cancer Research UK

Medical Research Council (UK)

Collaborators

Genkyotex - the company that owns Setanaxib (https://www.genkyotex.com/en/)

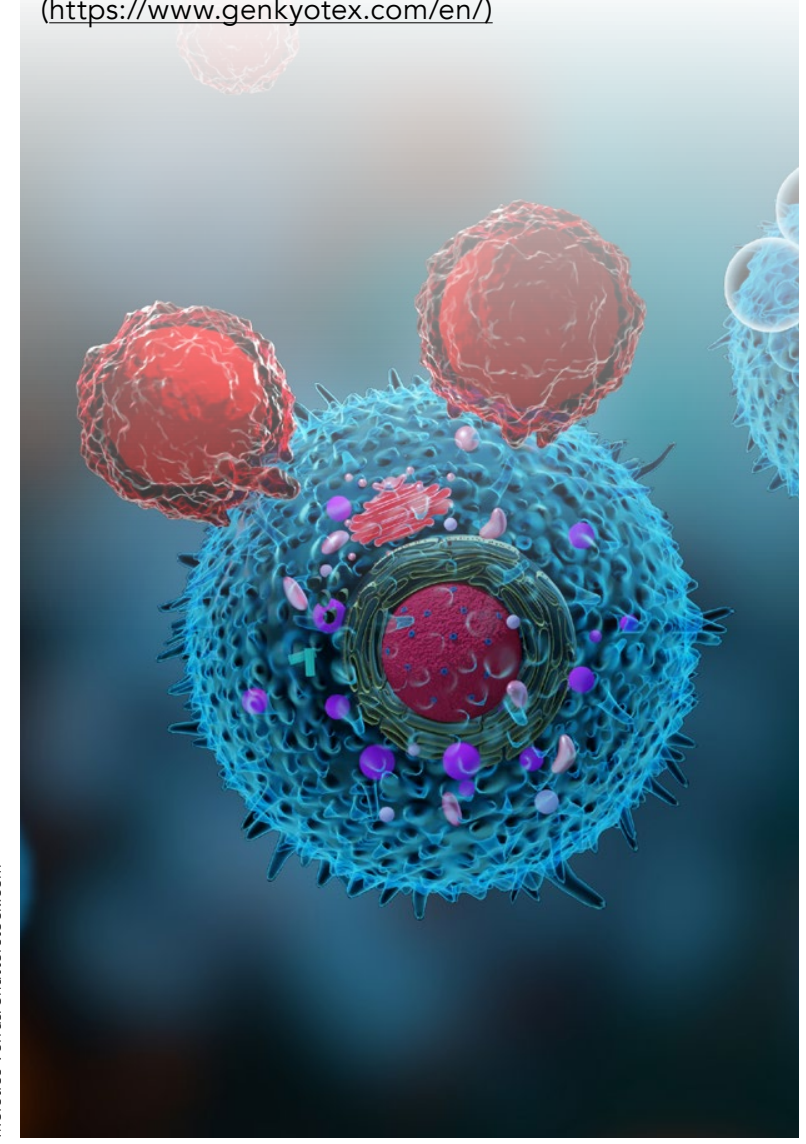

\section{References}

Hanley CJ, et al. 2018. Targeting the Myofibroblastic Cancer-Associated Fibroblast Phenotype Through Inhibition of NOX4. J Natl Cancer Inst. 110(1). doi 10.1093/jnci/djx121

Ford K, et al. 2020. NOX4 Inhibition Potentiates Immunotherapy by Overcoming Cancer-Associated Cancer Reserch doi: 10.1158/0008-5472.CAN-19-3158 Sahai $E$, et al. 2020. A framework for advancing our Reviews Cancer https://doi.org/10.1038/s41568-019-0238-1

Hanley CJ, et al. 2020. Spatially discrete signalling niches regulate fibroblast heterogeneity in human short/2020.06.08.134270v1

\section{Personal Response}

Are there other potential targets for blocking the

II TGF- $\beta$ signalling is central to CAF differentiation and ToF- $\beta 1$-neutralising antibodies can promote response targeting TGF- $\beta$ did not normalise the CAF phenotype or reduce CAF levels in tumours. Moreover, TGF- $\beta 1$ is a pleiotropic cytokine with roles in tissue homeostasis and tumour suppression, and global TGF- $\beta 1$ targeting is therefore a potentially risky strategy. Identifying mevasion could be a therapeutic strategy. CXC12 expression by CAF can mediate immunotherapy resistance, and inhibiting its receptor (CXCR4) has been
shown to improve response to checkpoint therapy.

Southanampton 